\title{
On the Application of Structured Sparse Model Selection to JPEG Compressed Images
}

\author{
Giovanni Maria Farinella and Sebastiano Battiato \\ Image Processing Laboratory, \\ Dipartimento di Matematica e Informatica, \\ Università degli Studi di Catania, \\ Viale A. Doria 6 - 95125 Catania, Italia \\ \{gfarinella, battiato\}@dmi.unict.it \\ http://iplab.dmi.unict.it
}

\begin{abstract}
The representation model that considers an image as a sparse linear combination of few atoms of a predefined or learned dictionary has received considerable attention in recent years. Among the others, the Structured Sparse Model Selection (SSMS) was recently introduced. This model outperforms different state-of-the-art algorithms in a number of imaging tasks (e.g., denoising, deblurring, inpainting). Despite the high denoising performances achieved by SSMS have been demonstrated, the compression issues has been not considered during the evaluation. In this paper we study the performances of SSMS under lossy JPEG compression. Experiments have shown that the SSMS method is able to restore compressed noisy images with a significant margin, both in terms of PSNR and SSIM quality measure, even though the original framework is not tuned for the specific task of compression. Quantitative and qualitative results pointed out that SSMS is able to perform both denoising and compression artifacts reduction (e.g., deblocking), by demonstrating the promise of sparse coding methods in application where different computational engines are combined to generate a signal (e.g., Imaging Generation Pipeline of single sensor devices).
\end{abstract}

Keywords: Sparse Coding, Inverse Problems, Compression, Denoising, Image Enhancement, Image Restoration.

\section{Introduction and Motivations}

Many imaging issues require to solve an inverse problem, that is, the problem of estimating an image $\boldsymbol{I}$ from a degraded version $\boldsymbol{J}$ which has been obtained through a non-invertible linear degradation operator $\boldsymbol{U}$, and further altered by an additive noise $\boldsymbol{w}$ :

$$
\boldsymbol{J}=\boldsymbol{U I}+\boldsymbol{w}
$$

Typical inverse problems in the context of image enhancement and restoration are Denoising (where $\boldsymbol{w}$ is the Gaussian white noise and $\boldsymbol{U}$ is neglected), Deblurring (where $\boldsymbol{U}$ is a convolution operator and $\boldsymbol{w}$ is the noise), 
Inpainting 1 (where $\boldsymbol{U}$ is a binary mask on the image and $\boldsymbol{w}$ is typically neglected) and Zooming (where $\boldsymbol{U}$ is a subsampling operator on a uniform grid and $\boldsymbol{w}$ is typically neglected).

Among the methods used to address the above inverse problems [1 8], sparse coding has been receiving considerable attention as it has shown promising results 9 16. Sparse coding is a method for modeling signals as sparse linear combinations of dictionary elements [17 -19]. The basic assumption of this model is that natural images admit a sparse decomposition in some redundant basis (or so-called dictionary). Each image patch is considered as a discrete array of positive numbers that can be generated by linear combinations of overcomplete bases set, where the natural statistics are captured by the fact that the vector of coefficients is sparse, so that to generate the image patch only few bases contribute. Image enhancement and restoration is performed through estimation of the sparse coefficient vectors, related the overcomplete bases set under consideration, which are useful to approximate the original image patches from the degraded version.

Despite sparse coding have been tested on different image enhancement problems, literature lacks of studies on application of sparse coding in presence of lossy compression or in general when unknown (or partially known) degradation processes have been applied simultaneously. This motivates the study reported in here.

The lossy compression process (i.e., JPEG compression [20]) attempts to eliminate redundant or unnecessary information. High compression factor could badly influence the quality of the final images highlighting undesirable effects. Blockbased coding, as in JPEG-compressed images, may produce a number of artifacts which give rise to undesirable visual patterns (e.g., blocking). A large number of approaches have been proposed in literature to reduce the undesirable effects of image compression at post-processing stage $21-23$. Some of them perform a post-filtering in shifted windows of image blocks analyzing the DCT or Wavelet domain during the smoothing procedure. The reduction of compression artifacts at post-processing stage is important to retain the benefits of the compression (for instance, lower transmission and storage costs). The big challenge is to obtain the best results in terms of standard quality measures with the smallest number of visual errors.

In this paper we present the results obtained employing the Structure Sparse Coding Model Selection (SSMS) [16] to restore compressed noisy images. SSMS has been demonstrated to be a powerful tool for different imaging issues (e.g., image denoising, deblurring and inpainting). Here we consider the problem of restoring gray and color images which have been altered by an additive Gaussian noise and further compressed with the lossy compression JPEG algorithm. The underlying ideas, that we start to address in this paper, is related to the fact that in presence of complex or unknown imaging pipelines, where different factors

${ }^{1}$ Demosaicking can be considered as a special case of the inpainting problem with regular subsampling on a specific uniform grid for each color channel, contaminated by an additive noise due to sensors characteristics. 
contribute to the degradation of the original signal (e.g., CFA subsampling, noise, compression, etc.), sparse coding methods could be adopted to restore the original signals.

Experimental results performed on the standard Kodak dataset2 show that the employed framework makes possible to recover information with a significant margin also in presence of high noise coupled with high compression factor, even though the original framework is not tuned for the specific task of compression. Quantitative and qualitative results pointed out that SSMS is able to perform both denoising and reduction of the blocking artifacts introduced by compression, by demonstrating the promise of sparse coding methods in the context under consideration.

The remainder of the paper is organized as follows: Section 2 introduces the sparse coding concepts, whereas Section 3 presents the SSMS framework for denoising. Section 4 reports the experiments and discusses the results obtained exploiting SSMS to restore compressed noisy images. Section [5] concludes the paper with avenues for further research.

\section{Sparse Coding for Image Enhancement and Restoration}

Sparse coding has emerged as powerful paradigm to describe signals based on the sparsity and redundancy of their representations [17 19]. For signals of a class $\Gamma \subset \Re^{N}$, this model suggests the existence of a dictionary $D \in \Re^{N \times K}$ which contains $K$ prototype signals $(|\Gamma| \gg K \geq N)$, also referred as atoms. The model assumes that for any signal $\boldsymbol{I} \in \Gamma$ there exists a sparse linear combination of atoms from $\boldsymbol{D}$ that approximates it well. When $K>N$ the dictionary is said to be redundant or overcomplete. The dictionary employed to sparsely represent the signals is usually learned from a dataset [19].

The sparse coding model has been successfully exploited in the contexts of image enhancement and restoration [9 16], where it is considered the state-ofthe-art in terms of both quantitative and qualitative results. In these contexts, images are decomposed into overlapping patches $I \in \Gamma$ of size $\sqrt{N} \times \sqrt{N}$. A patch is assumed to be sparsely represented in an overcomplete dictionary $\boldsymbol{D}=\left\{\boldsymbol{d}_{1}, \ldots, \boldsymbol{d}_{K}\right\} \subset \Gamma$ :

$$
\boldsymbol{I}=\boldsymbol{I}_{\boldsymbol{\Lambda}}+\boldsymbol{e}=\sum_{m=1}^{M} a_{m} \boldsymbol{d}_{m}^{\boldsymbol{\Lambda}}+\boldsymbol{e}
$$

where $\boldsymbol{d}_{m}^{\mathbf{\Lambda}} \in \boldsymbol{\Lambda} \subseteq \boldsymbol{D}, M=|\boldsymbol{\Lambda}| \leq K$ and the approximation error $\|\boldsymbol{e}\|^{2} \ll\|\boldsymbol{I}\|^{2}$. A sparse approximation $\widetilde{\boldsymbol{I}}=\sum_{m=1}^{M} \widetilde{a}_{m} \boldsymbol{d}_{m}^{\boldsymbol{\Lambda}}$ of $\boldsymbol{I}$ is obtained with a basis pursuit algorithm which minimizing a Lagrangian penalized by a sparse $l^{1}$ norm:

$$
\widetilde{\boldsymbol{a}}=\arg \min _{\boldsymbol{a}}\left\|\boldsymbol{I}-\sum_{k=1}^{K} a_{k} \boldsymbol{d}_{k}\right\|^{2}+\lambda\|\boldsymbol{a}\|_{1} .
$$

$\overline{2}$ The Kodak dataset is available at the http://rok.us/graphics/kodak/ 
In a typical inverse problem the aim is to estimate $\boldsymbol{I}$ from a degraded version

$$
\boldsymbol{J}=\boldsymbol{U} \boldsymbol{I} \boldsymbol{w}
$$

which has been obtained through a non-invertible linear degradation operator $\boldsymbol{U}$, and further altered by an additive noise $\boldsymbol{w}$. Taking into account the Equation (2) the degraded image $\boldsymbol{J}$ can be written as

$$
\boldsymbol{J}=\sum_{m=1}^{M} a_{m} \boldsymbol{U} \boldsymbol{d}_{m}^{\mathbf{\Lambda}}+\boldsymbol{e}^{\prime},
$$

with $\boldsymbol{e}^{\prime}=\boldsymbol{U} \boldsymbol{e}+\boldsymbol{w}$. This means that the degraded image $\boldsymbol{J}$ is well approximated by using the same coefficients $\boldsymbol{a}$ that are useful to sparsely approximate $\boldsymbol{I}$ in Equation (3) together with the transformed dictionary $\boldsymbol{U D}=\left\{\boldsymbol{U} \boldsymbol{d}_{1}, \ldots, \boldsymbol{U} \boldsymbol{d}_{K}\right\}$. The inverse problem of estimating an approximation of $\boldsymbol{I}$ from the degraded version $\boldsymbol{J}$ is hence solved by replacing the original dictionary $\boldsymbol{D}$ with the transformed one $\boldsymbol{U D}$ in the Equation (3).

\section{Structured Sparse Model Selection}

The degree of freedom in selecting the few atoms of the dictionary used to approximate $\boldsymbol{I}$ is exponentially large. This fact leads to unstable signal estimation. The Structured Sparse Model Selection (SSMS) put "structure" in sparsity to stabilize the estimation [16. SSMS is defined with a dictionary $\boldsymbol{D}$ composed by $H$ sub-dictionaries $\boldsymbol{B}^{1}, \ldots, \boldsymbol{B}^{H}$, each being an orthogonal basis. An image patch $\boldsymbol{I} \in \Re^{N}$ is assumed to be well approximated in one of these sub-dictionaries:

$$
\boldsymbol{I}=\sum_{m=1}^{M}\left\langle\boldsymbol{I}, \boldsymbol{b}_{m}^{h_{0}}\right\rangle \boldsymbol{b}_{m}^{h_{0}}+\boldsymbol{e}^{h_{0}},
$$

where $h_{0}$ is the index of the sub-dictionary that best approximate $\boldsymbol{I}$ in Equation (6) . The best basis $\boldsymbol{B}^{h_{0}}$ is selected by maximizing the projection energy on $\boldsymbol{B}^{h}=\left\{\boldsymbol{b}_{1}^{h}, \boldsymbol{b}_{2}^{h}, \ldots, \boldsymbol{b}_{M}^{h}\right\}$ over all the orthogonal bases $\boldsymbol{B}^{h} \in \boldsymbol{D}$ :

$$
h_{0}=\arg \min _{h} \sum_{m=1}^{M}\left|\left\langle\boldsymbol{I}, \boldsymbol{b}_{m}^{h}\right\rangle\right|^{2} .
$$

In this model the sub-dictionaries are initialized with Principal Component Analysis (PCA) over syntetic edge patterns of size $\sqrt{N} \times \sqrt{N}$. The edge patterns are grouped taking into account various orientations (Figure 1). For each orientation, the PCA over the relative syntetic edge patterns is computed and a basis is obtained. For each basis only the first $\sqrt{N}$ eigenvectors are retained, whereas the others are discarded due their negligible corresponding eigenvalues. The first eigenvector is replaced with the DC component. 


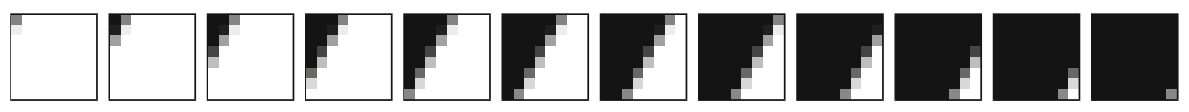

Fig. 1. Syntetic edge patterns with orientation $30^{\circ}$

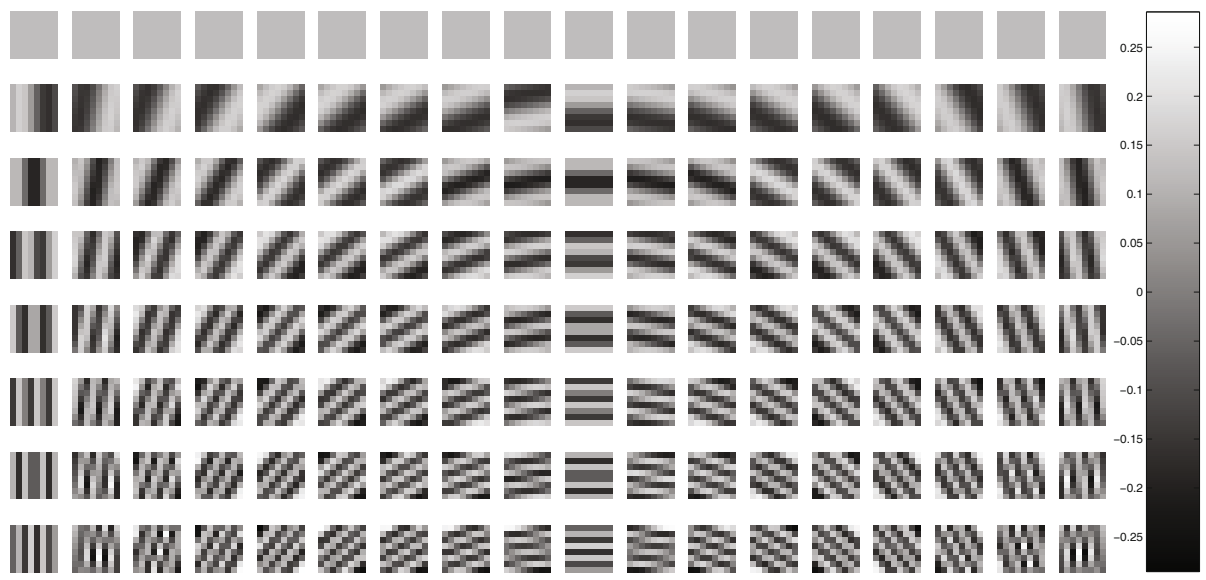

Fig. 2. The dictionary obtained through PCA on syntetic edge patterns grouped taking into account different orientations. Columns correspond to a specific subdictionary (one for each orientation). Each basis is composed by eight atoms (rows). The forth column is related the basis obtained considering the syntetic edge patterns in Figure 1

Figure 2 shows the sub-dictionaries computed on the syntetic edge patterns of size $8 \times 8$ and 18 different orientations (from $0^{\circ}$ to $170^{\circ}$ with a step of $10^{\circ}$ ). The sub-dictionaries are further adapted to the image of interest by applying the PCAs over the image patches, grouped following the model selection. First, the SSMS assign a model $h$ to each image patch, then each basis $\boldsymbol{B}^{h}$ is adapted to the image by recalculating the PCA with all the image patch that have been assigned to the model $h$.

In case of denoising task, where images are degraded with Gaussian noise $\boldsymbol{w}$ of variance $\sigma^{2}$, the aim is to estimate $\boldsymbol{I}$ from the degraded patch $\boldsymbol{J}=\boldsymbol{I}+\boldsymbol{w}$. The final patch approximation computed by SSMS model is obtained with a thesholding estimator in the best basis $\boldsymbol{B}^{h_{0}}$ :

$$
\widetilde{\boldsymbol{I}}=\sum_{\boldsymbol{b}_{m}^{h_{0}} \in \Lambda}\left\langle\boldsymbol{J}, \boldsymbol{b}_{m}^{h_{0}}\right\rangle \boldsymbol{b}_{m}^{h_{0}} .
$$

where $\Lambda=\left\{\boldsymbol{b}_{m}^{h_{0}}:\left|\left\langle\boldsymbol{J}, \boldsymbol{b}_{m}^{h_{0}}\right\rangle\right|>T\right\}$. 


\section{Experimental Results}

Experiments on restoring compressed noisy images have been carried out with the 24 standard benchmark images of the Kodak database. Each test involved an image of the dataset considered in grayscale or color, corrupted with additive Gaussian noise $(\sigma=5,10,20)$, and finally compressed (compression quality = Uncompressed, 100, 75, 50, 25, 15) through the JPEG Matlab engine. The SSMS was employed with patches of size $N=8 \times 8, H=18$ sub-dictionary computed by considering syntetic edge patterns at different orientations (from $0^{\circ}$ to $170^{\circ}$ with a step of $10^{\circ}$ ), size of each sub-dictionary $M=8$, and threshold as suggested in [16] $(T=3 \sigma)$. In practical use, a preliminary noise estimation phase $([25-27])$ could help to properly set the threshold $T$.

The peak signal-to-noise ratio (PSNR) and the structural similarity index (SSIM) [24] are used as performance measure in our quantitative evaluation. The PSNR is considered to assess the quality of reconstruction of the lossy compressed noisy images, whereas SSIM index is useful to assess the quality taking into account the human eye perception.

Table 1 and Table 2 report the quantitative results considering the images in grayscale. For each couple of parameters ( $\sigma$, compression quality), the reported PSNR and SSIM values are obtained averaging over the PSNR and SSIM results with respect to the 24 images of the Kodak dataset. Experiments point out that SSMS model leads to recover from $0.5545 \mathrm{~dB}(\sigma=5$, compression quality $=15)$ to $4.9879 \mathrm{~dB}(\sigma=20$, compression quality $=$ Uncompressed $)$ in terms of PSNR, with a gain from 0.0235 to 0.4299 in terms of SSIM. Visual inspection of the images reported in Figure 5] and Figure [6 is useful to assess the quality of the results. Note that in addition to the significant margin obtained in terms of PSNR and SSIM, visual results show that the model leads to remove some undesirable artifacts introduced by JPEG compression, even though the framework is not tuned for the specific task of compression.

Table 3 and Table 4 report the quantitative results obtained considering the images in the RGB color space. For each combination of parameters (color channel, compression quality and $\sigma$ ), the PSNR and SSIM values are obtained averaging over the PSNR and SSIM results related the 24 images of the Kodak dataset. The average gain in terms of PSNR and SSIM for each color channel at different $\sigma$ and compression quality is reported in Figure 3 and Figure 4 , whereas in Figure 7 and Figure 8 shown the original images and the restored ones.

Since JPEG compression is performed in $\mathrm{YCbCr}$ color domain, we have further tested the performance of SSMS to restore compressed noisy images taking into account that color space (without chromatic subsampling). In Table 5 and Table 6] are reported the results obtained restoring an image belonging to the test dataset under consideration, whereas in Figure 9 is shown a particular of both, the input image and the restored one.

Quantitative and qualitative results confirm that SSMS is able to perform denoising as well as compression artifacts reduction (i.e., deblocking), hence demonstrating the promise of sparse coding methods to restore signals which have been corrupted by different combined non-invertible factors. 
Table 1. Quantitative evaluation through PSNR measure on the Kodak dataset considering images in grayscale

\begin{tabular}{|c|c|c|c|c|c|c|c|}
\hline \multirow{3}{*}{ Average PSNR Gray Images } & \multicolumn{9}{c|}{$\sigma$} & \multicolumn{2}{c|}{$\mathbf{2 0}$} \\
\cline { 3 - 9 } \multicolumn{1}{|c|}{} & \multicolumn{2}{|c|}{$\mathbf{5}$} & \multicolumn{2}{c|}{$\mathbf{1 0}$} & \multicolumn{2}{c|}{} \\
\cline { 2 - 8 } & Corrupted & Restored & Corrupted & Restored & Corrupted & Restored \\
\hline \multirow{4}{*}{ Compression Quality } & $\mathbf{1 5}$ & 34.0468 & 34.6012 & 33.1735 & 34.4414 & 30.5417 & 33.5587 \\
\cline { 2 - 8 } & $\mathbf{2 5}$ & 34.8751 & 35.6358 & 33.1437 & 35.1633 & 30.3355 & 34.2415 \\
\cline { 2 - 8 } & $\mathbf{5 0}$ & 35.7431 & 37.0212 & 32.9187 & 35.9523 & 29.4379 & 33.9803 \\
\cline { 2 - 8 } & $\mathbf{7 5}$ & 36.4144 & 38.4877 & 31.9832 & 36.5222 & 29.1156 & 34.0308 \\
\cline { 2 - 8 } & $\mathbf{1 0 0}$ & 37.1075 & 40.7517 & 32.0141 & 36.9737 & 29.2979 & 34.2741 \\
\cline { 2 - 8 } & Uncompressed & 37.1645 & 40.7581 & 32.0392 & 36.9838 & 29.3047 & 34.2926 \\
\hline
\end{tabular}

Table 2. Quantitative evaluation through SSIM measure on the Kodak dataset considering images in grayscale

\begin{tabular}{|c|c|c|c|c|c|c|c|}
\hline \multirow{3}{*}{\multicolumn{2}{|c|}{ Average SSIM Gray Images }} & \multicolumn{6}{|c|}{$\sigma$} \\
\hline & & \multicolumn{2}{|c|}{5} & \multicolumn{2}{|c|}{10} & \multicolumn{2}{|c|}{20} \\
\hline & & Corrupted & Restored & Corrupted & Restored & Corrupted & Restored \\
\hline \multirow{6}{*}{ Compression Quality } & 15 & 0.8118 & 0.8352 & 0.7647 & 0.8256 & 0.5407 & 0.7812 \\
\hline & 25 & 0.8498 & 0.8749 & 0.7512 & 0.8533 & 0.5687 & 0.8841 \\
\hline & 50 & 0.8703 & 0.9098 & 0.7298 & 0.8794 & 0.4190 & 0.8041 \\
\hline & 75 & 0.8766 & 0.9326 & 0.6627 & 0.8930 & 0.3779 & 0.8077 \\
\hline & 100 & 0.8617 & 0.9526 & 0.6573 & 0.9018 & 0.4022 & 0.8186 \\
\hline & Uncompressed & 0.8630 & 0.9524 & 0.6585 & 0.9017 & 0.4027 & 0.8187 \\
\hline
\end{tabular}

Table 3. Quantitative evaluation through PSNR measure on the Kodak dataset considering images in the RGB color space

\begin{tabular}{|c|c|c|c|c|c|c|c|}
\hline \multirow{3}{*}{\multicolumn{2}{|c|}{ Average PSNR - Channel R }} & \multicolumn{6}{|c|}{$\sigma$} \\
\hline & & \multicolumn{2}{|c|}{5} & \multicolumn{2}{|c|}{10} & \multicolumn{2}{|c|}{20} \\
\hline & & Corrupted & Restored & Corrupted & Restored & Corrupted & Restored \\
\hline \multirow{6}{*}{ Compression Quality } & 15 & 32.9902 & 33.3342 & 32.7338 & 33.3179 & 31.5153 & 32.8581 \\
\hline & 25 & 34.2075 & 34.6793 & 33.6011 & 34.4384 & 31.4757 & 33.4783 \\
\hline & 50 & 35.4551 & 36.1144 & 34.0996 & 35.4205 & 30.9785 & 33.7740 \\
\hline & 75 & 36.6877 & 37.6297 & 34.0933 & 36.1304 & 30.2216 & 33.9218 \\
\hline & 100 & 39.4043 & 40.5143 & 34.1726 & 36.7819 & 30.3628 & 34.0484 \\
\hline & Uncompressed & 37.1565 & 40.6088 & 32.0294 & 36.8196 & 29.2926 & 34.0492 \\
\hline
\end{tabular}

\begin{tabular}{|c|c|c|c|c|c|c|c|}
\hline \multirow{3}{*}{\multicolumn{2}{|c|}{ Average PSNR - Channel G }} & \multicolumn{6}{|c|}{$\sigma$} \\
\hline & & \multicolumn{2}{|c|}{5} & \multicolumn{2}{|c|}{10} & \multicolumn{2}{|c|}{20} \\
\hline & & Corrupted & Restored & Corrupted & Restored & Corrupted & Restored \\
\hline \multirow{6}{*}{ Compression Quality } & 15 & 33.6942 & 34.1499 & 33.3616 & 34.0998 & 31.8656 & 33.4347 \\
\hline & 25 & 34.7088 & 35.2838 & 34.0181 & 34.9745 & 31.6331 & 33.7891 \\
\hline & 50 & 36.0809 & 36.8430 & 34.4845 & 35.9155 & 31.1180 & 34.0652 \\
\hline & 75 & 37.3821 & 38.4397 & 34.4313 & 36.5933 & 30.2989 & 34.1409 \\
\hline & 100 & 39.7078 & 40.9313 & 34.1405 & 36.9259 & 30.3353 & 34.1864 \\
\hline & Uncompressed & 37.1648 & 40.6397 & 32.0526 & 36.8594 & 29.3193 & 34.1325 \\
\hline
\end{tabular}

\begin{tabular}{|c|c|c|c|c|c|c|c|}
\hline \multirow{3}{*}{\multicolumn{2}{|c|}{ Average PSNR - Channel B }} & \multicolumn{6}{|c|}{$\sigma$} \\
\hline & & \multicolumn{2}{|c|}{5} & \multicolumn{2}{|c|}{10} & \multicolumn{2}{|c|}{20} \\
\hline & & Corrupted & Restored & Corrupted & Restored & Corrupted & Restored \\
\hline \multirow{6}{*}{ Compression Quality } & 15 & 32.8844 & 33.2351 & 32.7255 & 33.3686 & 31.7672 & 33.3061 \\
\hline & 25 & 33.9941 & 34.4873 & 33.5374 & 34.4881 & 31.7236 & 34.0112 \\
\hline & 50 & 35.2433 & 35.9493 & 34.0432 & 35.5168 & 31.2101 & 34.3547 \\
\hline & 75 & 36.5143 & 37.5120 & 34.1305 & 36.3551 & 30.4399 & 34.5442 \\
\hline & 100 & 39.3584 & 40.4643 & 34.3871 & 37.0467 & 30.6662 & 34.6490 \\
\hline & Uncompressed & 37.1969 & 40.6160 & 32.0970 & 36.9827 & 29.3793 & 34.5623 \\
\hline
\end{tabular}


Table 4. Quantitative evaluation through SSIM measure on the Kodak dataset considering images in the RGB color space

\begin{tabular}{|c|c|c|c|c|c|c|c|}
\hline \multirow{3}{*}{\multicolumn{2}{|c|}{ Average SSIM - Channel R }} & \multicolumn{6}{|c|}{$\sigma$} \\
\hline & & \multicolumn{2}{|c|}{5} & \multicolumn{2}{|c|}{10} & \multicolumn{2}{|c|}{20} \\
\hline & & Corrupted & Restored & Corrupted & Restored & Corrupted & Restored \\
\hline \multirow{6}{*}{ Compression Quality } & 15 & 0.7953 & 0.8155 & 0.7835 & 0.8106 & 0.6831 & 0.7757 \\
\hline & 25 & 0.8431 & 0.8604 & 0.8119 & 0.8447 & 0.6556 & 0.7943 \\
\hline & 50 & 0.8836 & 0.9009 & 0.8194 & 0.8743 & 0.6102 & 0.8085 \\
\hline & 75 & 0.9059 & 0.9270 & 0.8059 & 0.8917 & 0.5334 & 0.8148 \\
\hline & 100 & 0.9222 & 0.9527 & 0.7828 & 0.9013 & 0.5454 & 0.8173 \\
\hline & Uncompressed & 0.8680 & 0.9528 & 0.6663 & 0.9028 & 0.4097 & 0.8196 \\
\hline
\end{tabular}

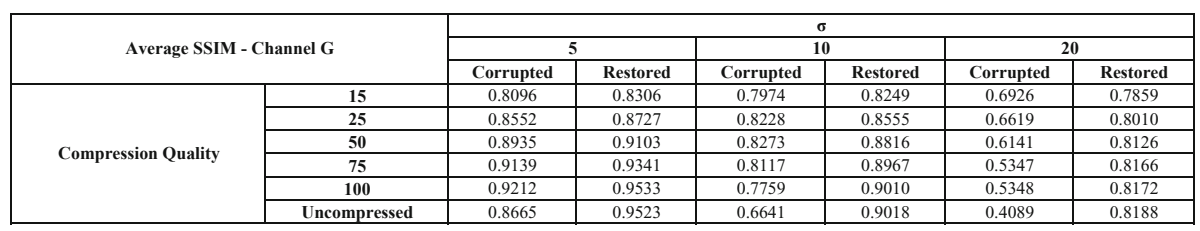

\begin{tabular}{|c|c|c|c|c|c|c|c|}
\hline \multirow{3}{*}{\multicolumn{2}{|c|}{ Average SSIM - Channel B }} & \multicolumn{6}{|c|}{$\sigma$} \\
\hline & & \multicolumn{2}{|c|}{5} & \multicolumn{2}{|c|}{10} & \multicolumn{2}{|c|}{20} \\
\hline & & Corrupted & Restored & Corrupted & Restored & Corrupted & Restored \\
\hline \multirow{6}{*}{ Compression Quality } & 15 & 0.7733 & 0.7955 & 0.7614 & 0.7927 & 0.6610 & 0.7622 \\
\hline & 25 & 0.8233 & 0.8434 & 0.7916 & 0.8304 & 0.6364 & 0.7828 \\
\hline & 50 & 0.8669 & 0.8872 & 0.8022 & 0.8624 & 0.5931 & 0.7966 \\
\hline & 75 & 0.8917 & 0.9157 & 0.7907 & 0.8811 & 0.5185 & 0.8014 \\
\hline & 100 & 0.9164 & 0.9449 & 0.7761 & 0.8896 & 0.5373 & 0.7992 \\
\hline & Uncompressed & 0.8604 & 0.9458 & 0.6514 & 0.8901 & 0.3949 & 0.8009 \\
\hline
\end{tabular}

Table 5. Average channels' PSNR obtained by considering the image in the YCbCr color space

\begin{tabular}{|c|c|c|c|c|c|c|c|}
\hline \multirow{3}{*}{$\begin{array}{l}\text { Average Channels' PSNR } \\
\text { (Kodim04) }\end{array}$} & \multirow[b]{3}{*}{$-2=2$} & \multicolumn{6}{|c|}{$\sigma$} \\
\hline & & \multicolumn{2}{|c|}{5} & \multicolumn{2}{|c|}{10} & \multicolumn{2}{|c|}{20} \\
\hline & & \begin{tabular}{|l|} 
Corrupted \\
\end{tabular} & \begin{tabular}{|l|} 
Restored \\
\end{tabular} & \begin{tabular}{|l|} 
Corrupted \\
\end{tabular} & Restored & Corrupted & Restored \\
\hline \multirow{5}{*}{ Compression Quality } & 15 & \begin{tabular}{|l|}
34.1606 \\
\end{tabular} & \begin{tabular}{l|l|}
34.8368 \\
\end{tabular} & \begin{tabular}{|l|}
33.9226 \\
\end{tabular} & 35.0148 & 32.4425 & 34.5508 \\
\hline & 25 & 35.6912 & 36.4566 & 34.9614 & 36.2366 & 32.1483 & 35.2412 \\
\hline & 50 & 37.2455 & 38.2577 & 35.3904 & 37.5269 & 31.4054 & 35.7042 \\
\hline & 75 & 38.4669 & 39.9343 & 35.0356 & 38.2294 & 30.4014 & 35.9062 \\
\hline & 100 & 39.7016 & 42.5318 & 34.3084 & 38.9809 & 30.4972 & 36.1308 \\
\hline
\end{tabular}

Table 6. Average channels' SSIM obtained by considering the image in the YCbCr color space

\begin{tabular}{|c|c|c|c|c|c|c|c|}
\hline \multirow{3}{*}{$\begin{array}{c}\text { Average Channels' SSIM } \\
\text { (Kodim04) }\end{array}$} & \multirow[b]{3}{*}{$=8$} & \multicolumn{6}{|c|}{$\sigma$} \\
\hline & & \multicolumn{2}{|c|}{5} & \multicolumn{2}{|c|}{10} & \multicolumn{2}{|c|}{20} \\
\hline & & Corrupted & Restored & Corrupted & Restored & Corrupted & Restored \\
\hline \multirow{5}{*}{ Compression Quality } & 15 & 0.8620 & 0.8897 & 0.8476 & 0.8920 & 0.7175 & 0.8639 \\
\hline & 25 & 0.8976 & 0.9198 & 0.8596 & 0.9123 & 0.6586 & 0.8790 \\
\hline & 50 & 0.9195 & 0.9426 & 0.8382 & 0.9294 & 0.5883 & 0.8904 \\
\hline & 75 & 0.9249 & 0.9557 & 0.8019 & 0.9358 & 0.4937 & 0.8927 \\
\hline & 100 & 0.9071 & 0.9681 & 0.7476 & 0.9417 & 0.5007 & 0.8960 \\
\hline
\end{tabular}




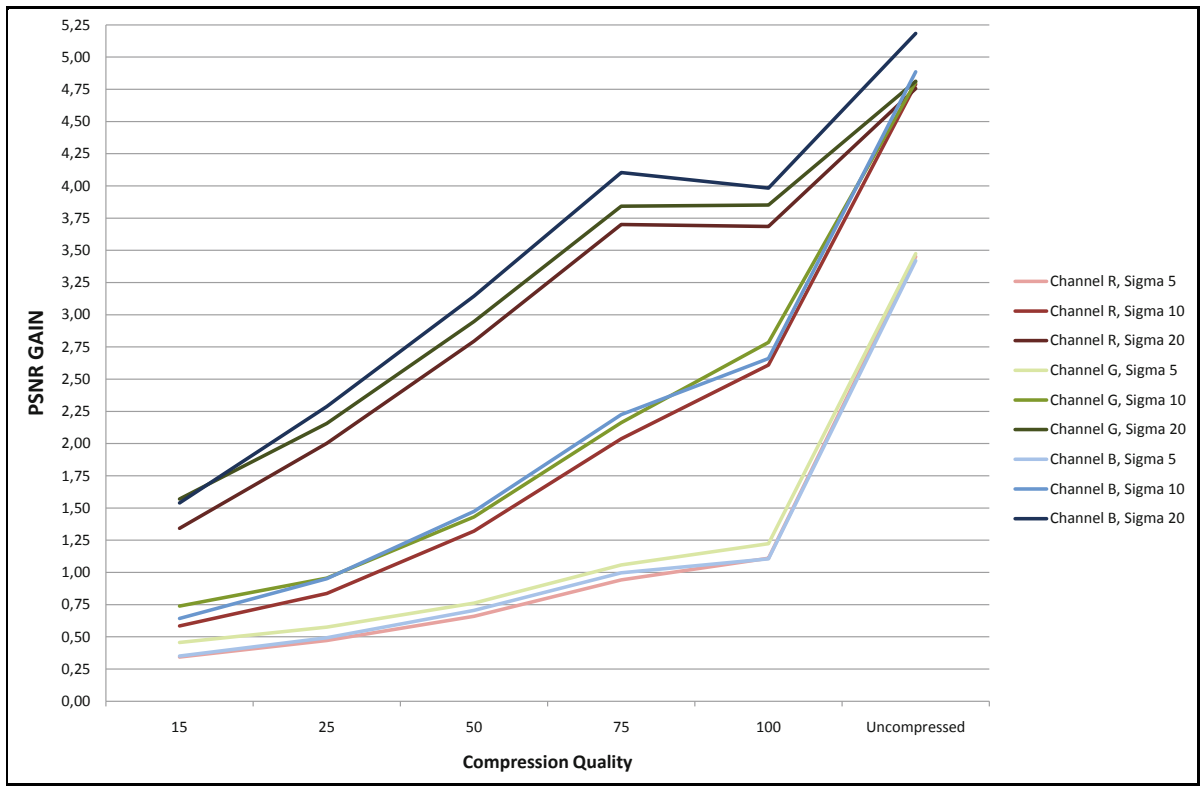

Fig. 3. Average gain in terms of PSNR considering the Kodak dataset in the RGB color space

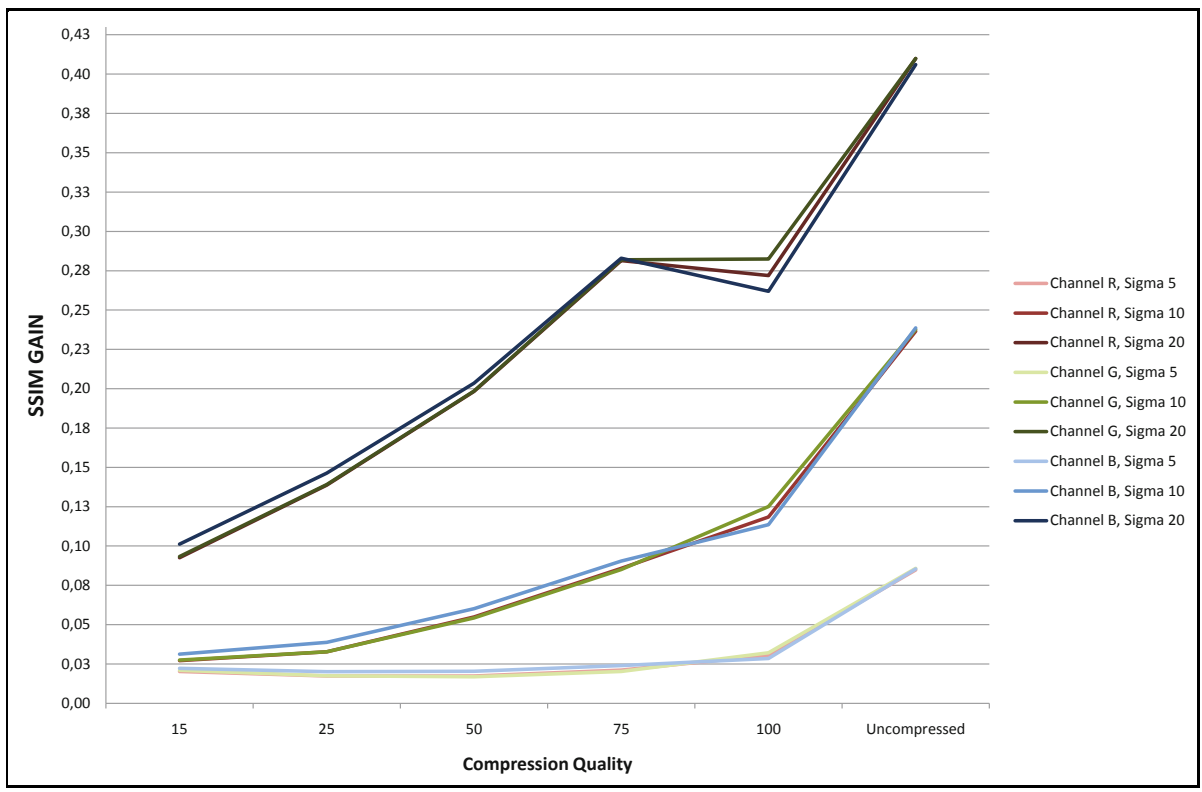

Fig. 4. Average gain in terms of SSIM considering the Kodak dataset in the RGB color space 


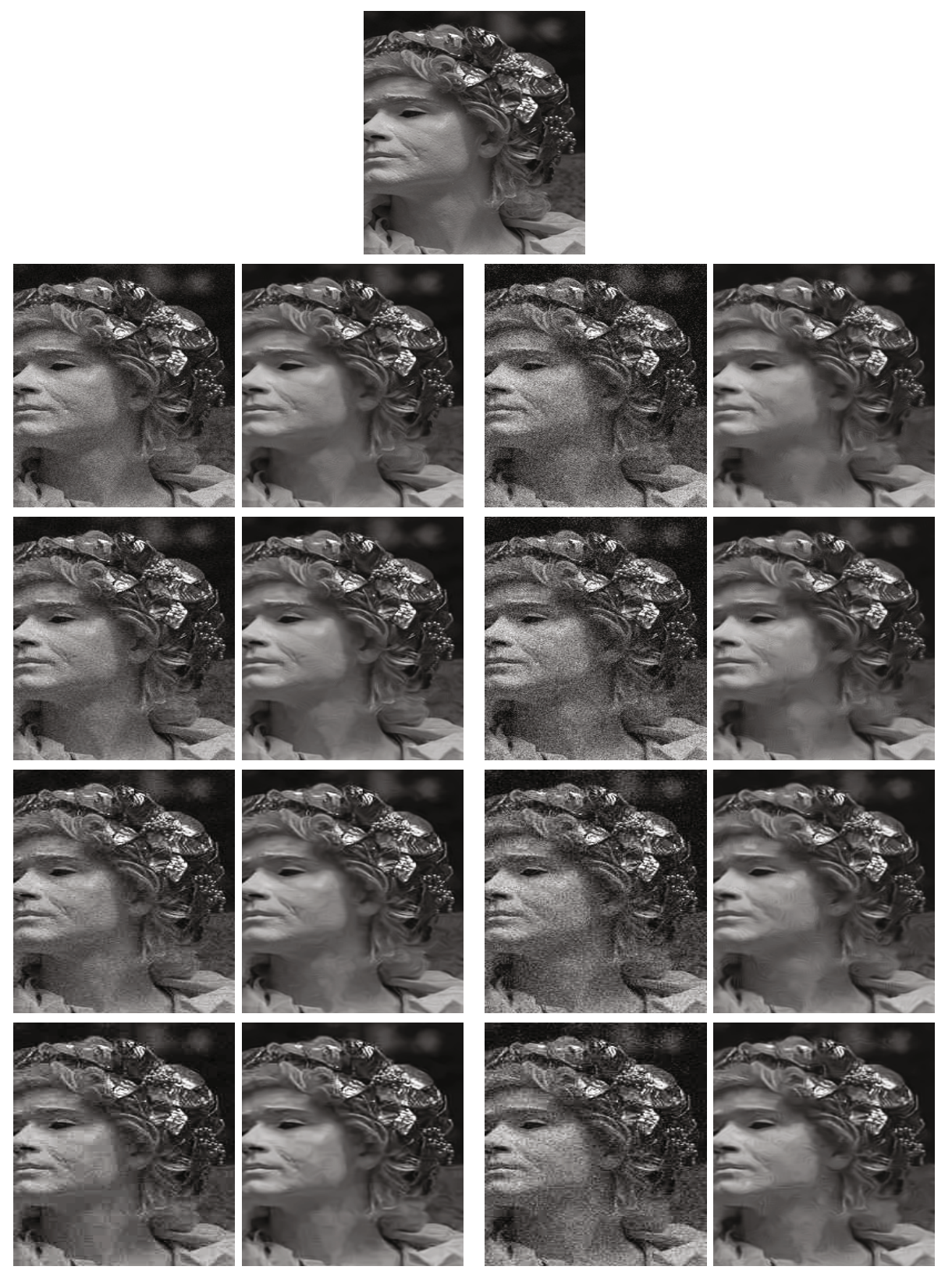

Fig. 5. Qualitative evaluation on grayscale images. The original image is shown at the top. Images altered by an additive Gaussian noise are shown in the odd columns (from left to right: $\sigma=10,20)$, whereas even columns correspond to the restored images. Rows are related the compression quality applied to the noisy image (from top to bottom: $75,50,25,15)$. The details are better seen by zooming on a computer screen. 

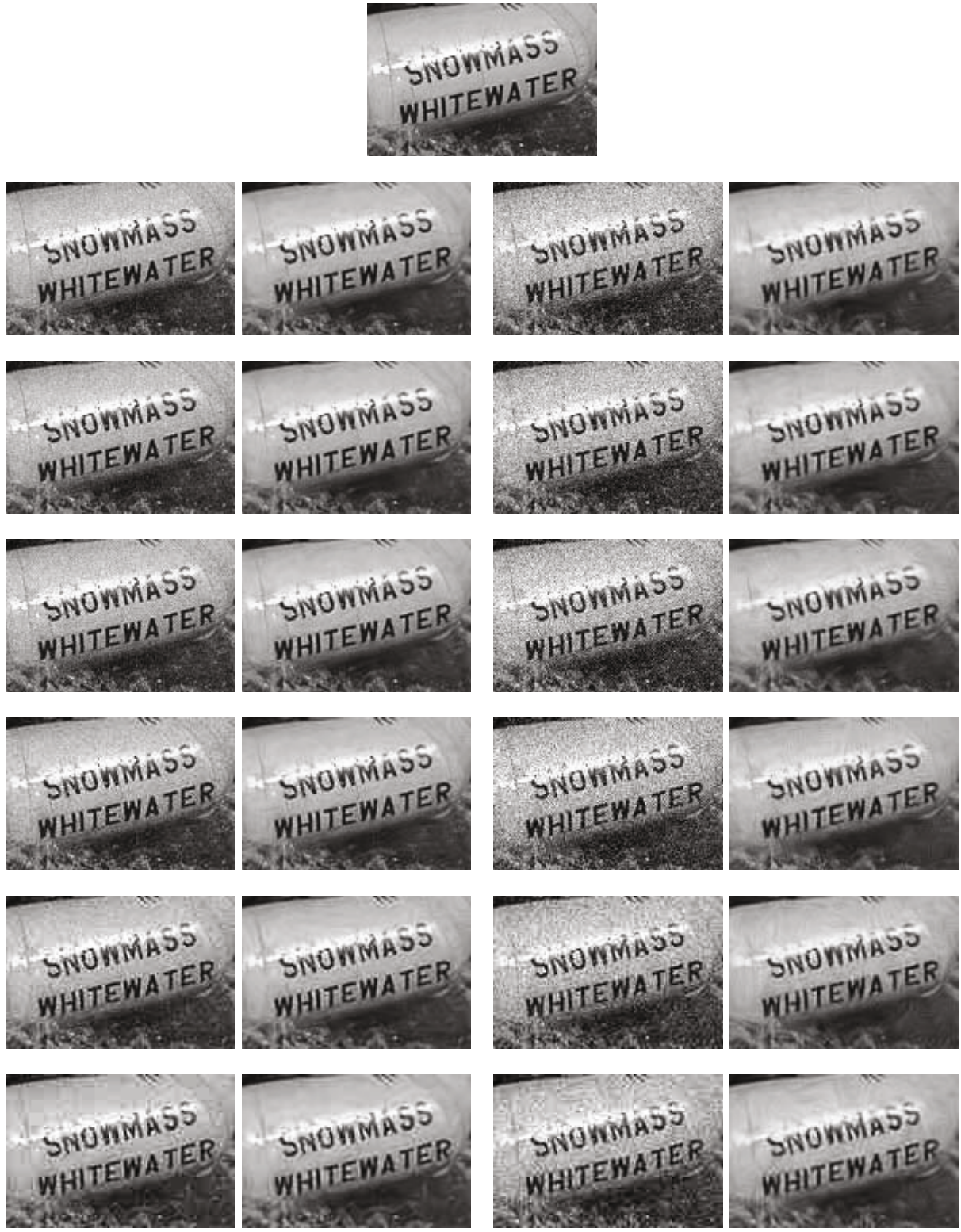

Fig. 6. Qualitative evaluation on grayscale images. The original image is shown at the top. Images altered by an additive Gaussian noise are shown in the odd columns (from left to right: $\sigma=10,20)$, whereas even columns correspond to the restored images. Rows are related the compression quality applied to the noisy image (from top to bottom: Uncompressed, 100,75,50,25,15). The details are better seen by zooming on a computer screen. 

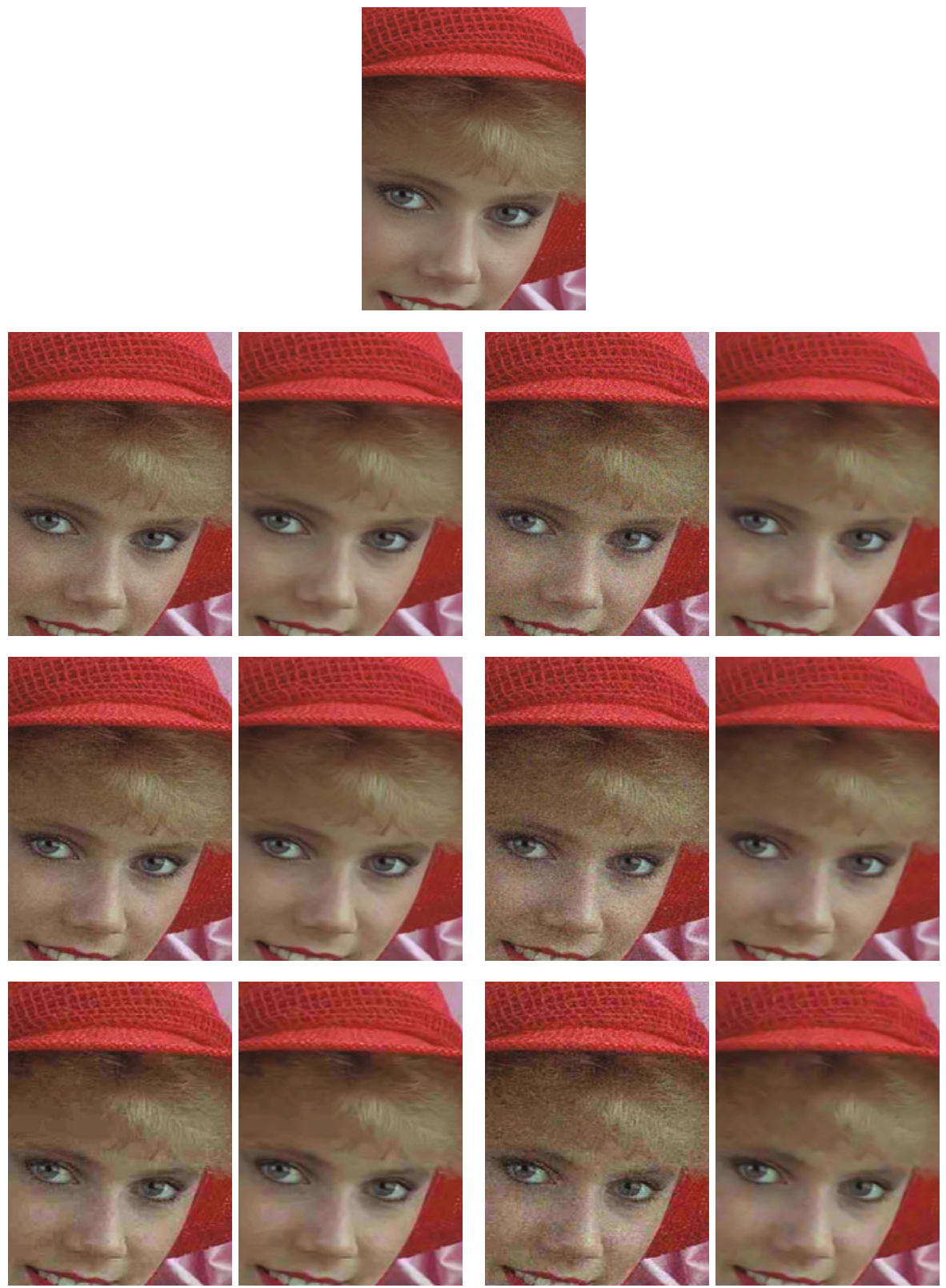

Fig. 7. Qualitative evaluation on RGB images. The original image is shown at the top. Images altered by an additive Gaussian noise are shown in the odd columns (from left to right: $\sigma=10,20)$, whereas even columns correspond to the restored images. Rows are related the compression quality applied to the noisy image (from top to bottom: $50,25,15)$. The details are better seen by zooming on a computer screen. 

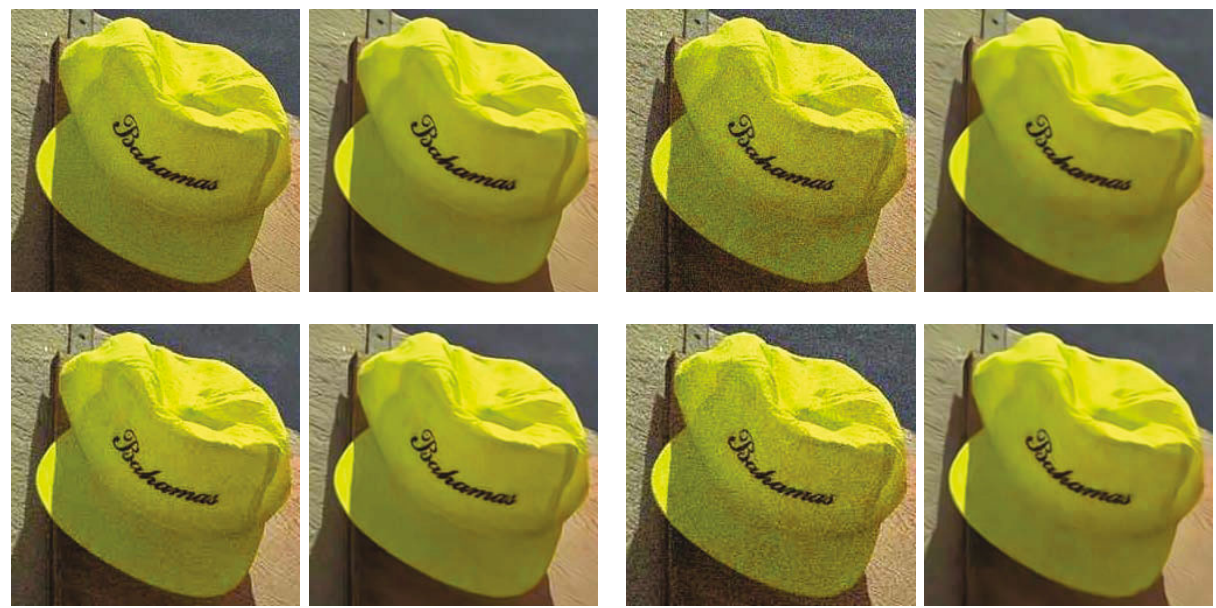

Fig. 8. Qualitative evaluation on RGB images. Images altered by an additive Gaussian noise are shown in the odd columns (from left to right: $\sigma=10,20$ ), whereas even columns correspond to the restored images. Rows are related the compression quality applied to the noisy image (from top to bottom: 50,25). The details are better seen by zooming on a computer screen.
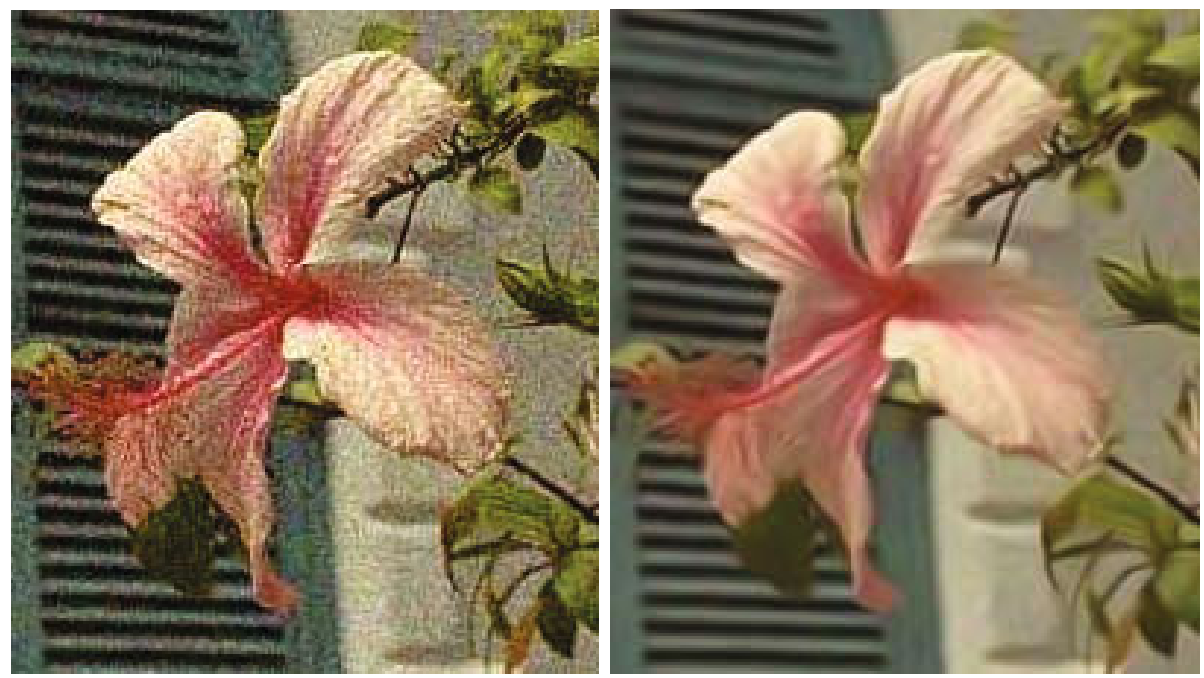

Fig. 9. Qualitative evaluation on YCbCr images. Left: corrupted image ( $\sigma=20$, Compression Quality $=50)$. Right: restored image. The details are better seen by zooming on a computer screen. 


\section{Conclusion and Future Works}

Sparse Coding has received considerable attention in recent years as model useful in different imaging problems. In this paper we have considered the problem of restoring information from lossy compressed noisy images by employing the Structured Sparse Model Selection (SSMS) approach. Although the method was not tuned for the specific task of compression, the experiments have shown that it is able to recover significant information obtaining good quantitative and qualitative performances. These preliminary results lead to push our future research in studying sparse coding methods which take into account the compression operator in modeling the representation of the image. Ad-hoc quality measures to assess the deblocking properties [28] should be taken into account in future works.

\section{References}

1. Bertalmío, M., Sapiro, G., Caselles, V., Ballester, C.: Image Inpainting. In: SIGGRAPH (2000)

2. Li, X., Orchard, M.T.: New edge-directed interpolation. IEEE Transactions on Image Processing 10(10), 1521-1527 (2001)

3. Battiato, S., Gallo, G., Stanco, F.: A Locally-Adaptive Zooming Algorithm for Digital Images. Image Vision and Computing Journal 11(20), 805-812 (2002)

4. Buades, A., Coll, B., Morel, J.-M.: A Non-Local Algorithm for Image Denoising. In: IEEE Computer Society Conference on Computer Vision and Pattern Recognition (2005)

5. Buades, A., Coll, B., Morel, J.M.: A review of image denoising algorithms, with a new one. Multiscale Modeling and Simulation 4(2), 490-530 (2006)

6. Battiato, S., Bosco, A., Bruna, A.R., Rizzo, R.: Noise Reduction for CFA Image Sensors Exploiting HVS behavior. Sensors Journal - MDPI Open Access - Special Issue on Integrated High-Performance Imagers 3(9), 1692-1713 (2009)

7. Joshi, N., Zitnick, C.L., Szeliski, R., Kriegman, D.J.: Image deblurring and denoising using color priors. In: Computer Vision and Pattern Recognition (2009)

8. Battiato, S., Guarnera, M., Messina, G., Tomaselli, V.: Recent patents on color demosaicing. Recent Patents on Computer Science 1(2), 194-207 (2008)

9. Aharon, M., Elad, M., Bruckstein, A.: K-SVD: An algorithm for designing overcomplete dictionaries for sparse representation. IEEE Transaction on Signal Processing 54(11), 4311-4322 (2006)

10. Elad, M., Aharon, M.: Image denoising via sparse and redundant representations over learned dictionaries. IEEE Transactions on Image Processing 54(12), 37363745 (2006)

11. Mairal, J., Elad, M., Sapiro, G.: Sparse representation for color image restoration. IEEE Transactions on Image Processing 17(1), 53-69 (2008)

12. Mairal, J., Bach, F., Ponce, J., Sapiro, G., Zisserman, A.: Discriminative Learned Dictionaries for Local Image Analysis. In: IEEE International Conference on Computer Vision and Pattern Recognition (2008)

13. Mairal, J., Bach, F., Ponce, J., Sapiro, G., Zisserman, A.: Non-local sparse models for image restoration. In: International Conference on Computer Vision (2009) 
14. Lou, Y., Bertozzi, A., Soatto, S.: Direct sparse deblurring. Technical Report, CAMUCLA (2009)

15. Fadili, M.J., Starck, J.L., Murtagh, F.: Inpainting and zooming using sparse representations. The Computer Journal 52(1) (2009)

16. Yu, G., Sapiro, G., Mallat, S.: Image Modeling and Enhancement via Structured Sparse Model Selection. In: IEEE International Conference on Image Processing (2010)

17. Olshausen, B.A., Field, D.J.: Sparse coding with an overcomplete basis set: A strategy employed by V1? Vision Research 37, 3311-3325 (1997)

18. Olshausen, B.A., Field, D.J.: Sparse Coding of Sensory Inputs. Current Opinion in Neurobiology 14, 481-487 (2004)

19. Mairal, J., Bach, F., Ponce, J., Sapiro, G.: Online dictionary learning for sparse coding. In: International Conference on Machine Learning (2009)

20. Wallace, G.K.: The JPEG still picture compression standard. Communications of the ACM 34(4) (1991)

21. Zhai, G., Zhang, W., Yang, X., Lin, W.: Efficient Image Deblocking Based on Post filtering in Shifted Windows. IEEE Transactions on Circuits and Systems for Video Technology 18(1), 122-126 (2008)

22. Zhai, G., Zhang, W., Yang, X., Lin, W., Xu, Y.: Efficient Deblocking With Coefficient Regularization, Shape-Adaptive Filtering, and Quantization Constraint. IEEE Transactions on Multimedia 10(5), 735-745 (2008)

23. Kim, J.: Adaptive Blocking Artifact Reduction using Wavelet-Based Block Analysis. IEEE Transactions on Consumer Electronics (55), 2 (2009)

24. Wang, Z., Bovik, A.C., Sheikh, H.R., Simoncelli, E.P.: Image quality assessment: From error visibility to structural similarity. IEEE Transactions on Image Processing 13(4), 600-612 (2004)

25. Foi, A., Trimeche, M., Katkovnik, V., Egiazarian, K.O.: Practical PoissonianGaussian Noise Modeling and Fitting for Single-Image Raw- Data. IEEE Transactions on Image Processing 17(10), 1737-1754 (2008)

26. Kim, Y.-H., Lee, J.: Image feature and noise detection based on statistical hypothesis tests and their applications in noise reduction. IEEE Transactions on Consumer Electronics 51(4), 1367-1378 (2005)

27. Bosco, A., Bruna, A., Giacalone, D., Battiato, S., Rizzo R.: Signal-Dependent Raw Image Denoising Using Image Sensor Characterization Via Multiple Acquisitions. In: SPIE Electronic Imaging 2010 - Digital Photography VI (2010)

28. Yim, C., Bovik, A.: Quality Assessment of De-blocked Images. IEEE Transaction on Image Processing 20(1), 88-98 (2011) 